\title{
Cirugía tubárica frente a reproducción asistida
}

\author{
Jaime Saavedra, Saavedra, $M D^{*}$ \\ Recibido: febrero 14/2002 - Revisado: marzo 4/2002 - Aceptado: mayo 30/2002
}

RESUMEN

Toda pareja infértil con factor tubo peritoneal comprometido debe ser estudiada en forma completa; esto incluye espermograma, niveles basales de FSH el día tres del ciclo, histerosalpingografía y laparoscopia diagnóstica.

Estos estudios son decisivos para determinar si la pareja se refiere al programa de cirugía tubárica reconstructiva o al programa de técnicas de reproducción asistida.

Las tasas acumulativas de embarazo en adhesiolisis de adherencias laxas (avasculares) están entre un 50 y un 60\%; en adherencias extensas (densas y vasculares) es de $0 \%$, por tanto estas pacientes deben ir a técnicas de reproducción asistida (FIV).

En el caso de fimosis tubárica, la fimbrioplastia por microcirugía presenta una tasa de embarazo del $60 \%$ frente a un 30\% por laparoscopia, por tanto para el manejo de esta patología se recomienda la microcirugía.

Las tasas de embarazo en caso de hidrosalpinx con daño tubárico mínimo y limitado (presencia de pliegues mucosos en la histerosalpingografía y menos de dos centímetros de diámetro) están entre un $28 \%$ por microcirugía y un $24 \%$ por laparoscopia; en caso de daño tubárico severo se debe realizar FIV.

Las tasas de embarazo clínico después de FIV-TE en pacientes con factor tubárico varían entre un 30.8 y un $12.8 \%$, dependiendo de la edad de la paciente, estas tasas son mejores en mujeres menores de 35 años y bajas en mujeres de 40 años y más.

Cuando se consideran las tasas acumulativas de embarazo después de cuatro ciclos de reproducción asistida frente a salpingoovariolisis, fimbrioplastia y salpingoneostomía, la primera da mejores resultados en todos los frentes. Sin embargo, podría ser un error pronosticar que la cirugía reconstructiva vaya a ser historia pronto. Si la cirugía es exitosa ésta ofrece la posibilidad de concebir en múltiples ciclos y lograr más embarazos en forma consecutiva.

El riesgo de embarazo múltiple es bajo después del tratamiento quirúrgico.

El factor económico también puede pesar en el momento de tomar una decisión.

Los resultados anteriores sugieren que las mujeres jóvenes, con factor tubo peritoneal leve o moderado, son candidatas primarias para la cirugía tubárica reconstructiva, mientras que mujeres mayores de 35 años o aquéllas con enfermedad severa o pelvis congelada se deben dirigir al programa de reproducción asistida.

Palabras clave: cirugía tubárica, adhesiolisis, fimbrioplastia, hidrosalpinx, reproducción asistida.

\footnotetext{
* Profesor titular, Departamento de Ginecología y Obstetricia, Universidad del Valle; jefe del Servicio de Ginecología y Obstetricia, Universidad del Valle; director Programa de Entrenamiento de Cirugía Endoscópica, Clínica los Andes, Cali, Colombia; director general del Centro de Biomedicina Reproductiva (Fecundar) Cali, Colombia.
} 


\section{SUMMARY}

Every infertile couple with tuboperitoneal factor must be completely studied; this includes semen analysis, basal levels of FSH on the third day of the cycle, histerosalpingography and diagnostic laparoscopic. These studies are important to determine if the couple should be offered fertility surgery or Assisted Reproductive Technology (ART).

The accumulative pregnancy rates in adhesiolysis of minimal (avascular, filmy) adhesions are between $50 \%$ and $60 \%$, to $0 \%$ births with extensive (dense, vascular) adhesions, therefore these patients must go to ART program.

In the case of tubaric phymosis the microsurgery for fimbrioplasty has a pregnancy rate of $60 \%$ versus a $30 \%$ by laparoscopy, so for the treatment of this pathology the microsurgery is recommended.

The pregnancy rates, in the case of hydrosalpinx with a small and minimum tubaric injured (presence of mucous folds in the histerosalpingography and less of 2 centimetres of diameter) are between a $28 \%$ by microsurgery and a $24 \%$ by laparoscopy, in case of a severed injured the patient, must go to ART.

The clinic pregnancy rates after IVF-TE with tubaric factor vary between a $30.8 \%$ and $12.8 \%$ depending on the age of patient; having better rates in women under 35 , and not good in women over 40 .

When we consider the cumulative pregnancy rates after four cycles of assisted reproduction against ovariosalpingolysis, fimbrioplasty or salpingoneostomy, then the former achieves better results on each front. Nevertheless it would be wrong to predict that reconstructive surgery will soon be history. First of all, if surgery is successful it offers the possibility of conceiving in multiple cycles and to achieve multiple consecutive pregnancies. The risk of multiple gestation is lower after surgical treatment.

Economic factors, on the other hand, can also influence decision-making.

The above results suggest that younger women with mild or moderate tubeperitoneal disturbance are primary candidates for tubal reconstructive surgery, while older patients with severe disease or frozen pelvis should be directed immediately to an assisted reproduction program.

Key words: Tubal surgery, salpingolysis, fimbrioplasty, salpingoneostomy, assisted reproduction.

En la década pasada hubo un gran progreso en el tratamiento de la infertilidad, especialmente en el campo de la tecnología de la reproducción asistida. Esta evolución ha dado como resultado un cambio en el manejo de la pareja infértil, colocando la cirugía pélvica o tubárica en la parte trasera de este manejo (1-2). Otras formas de cirugía pélvica seguirán siendo prevalentes en mujeres en edad reproductiva (p. ej. cirugía de endometriosis, cistectomía ovárica, miomectomía)

Se han publicado muchos artículos relacionados con la microcirugía en el campo de la infertilidad tubárica. La laparoscopia quirúrgica ha intentado reemplazar a la microcirugía, siendo ésta más bien nueva en esta área. Hay pocos datos prospectivos conocidos relacionados con el resultado de fertilidad después de cirugía tubárica laparoscópica reconstructiva; las tasas crudas retrospectivas de embarazos después de laparoscopia, y después de microcirugía fluctúan alrededor del 30\%. La patología tubárica que distorsiona la captación del óvulo se ha incrementado.

La infertilidad no se debe a menudo a un solo factor; las indicaciones para fertilización in vitro (FIV), que se desarrollaron inicialmente para tratar mujeres con patología tubárica no operable, se han extendido; particularmente la introducción de la inyección intra citoplasmática (ICSI) para tratar casos severos de infertilidad masculina a añadido nuevas dimensiones al tratamiento de la infertilidad.

El propósito de este artículo es dar al lector una comprensión del papel de la FIV en el manejo de la enfermedad tubárica incluyendo factores pronósticos que tienen un impacto significativo sobre los resultados del éxito. 
Una comprensión de las tasas de éxito, los riesgos, las complicaciones de la cirugía tubárica y del FIV, van a dar al médico una guía apropiada y a la pareja infértil que sufre de una enfermedad tubárica unas expectativas reales de su problema.

La decisión adecuada con relación a un manejo quirúrgico frente a técnicas de reproducción asistida requiere del conocimiento de las alternativas disponibles, facilitando de este modo un consejo a la pareja infértil.

\section{INFERTILIDAD TUBÁRICA}

La disrupción de la anatomía y función tubárica normal corresponde a un $40 \%$ de la infertilidad femenina (3). La enfermedad pélvica inflamatoria, la infección intraabdominal (apendicitis), la cirugía pélvica $\mathrm{y}$, menos comúnmente, la endometriosis a menudo resultan en oclusión tubárica distal junto a grados variables de dilatación y daño de la mucosa (4). Entre 1960 y 1980 la infertilidad como causa de procesos infecciosos aumentó en un factor de 1,6 veces, haciendo de la enfermedad pélvica inflamatoria la causa más común de infertilidad (5). Por otro lado, la obstrucción tubárica media se ve más frecuentemente después de la esterilización quirúrgica o la reseción tubárica segmentaria de un embarazo ectópico (6). Un proceso infeccioso rara vez produce una oclusión tubárica media.

La etiología de la oclusión tubárica proximal (unión uterotubárica) no es clara. Ésta se estima entre un $10-25 \%$. Se ha reportado la presencia de tapones amorfos que obstruyen la luz tubárica (7); las adherencias peritubárica y periováricas sin producir una verdadera oclusión, pueden agravar la infertilidad (8-9). La movilidad de la trompa y el ovario se pueden dañar por estas adherencias, dando como resultado una pobre captación del óvulo. Las adherencias no son un problema aislado ya que probablemente están asociadas con daño significativo del endotelio tubárico; Gomel (8), Bowman y Cook (10) confirmaron esto. Ellos hallaron una fuerte correlación entre el grado de daño de la mucosa tubárica confirmada mediante salpingoscopia y la extensión de las adherencias pélvicas en pacientes con historia de enfermedad pélvica inflamatoria.
Esta correlación no ha sido demostrada en pacientes con enfermedad adherencial como resultado de endometriosis.

El objetivo de la cirugía en los casos de infertilidad tubárica es restaurar la anatomía salpingoovárica y el lumen tubárico, de tal forma que la fertilización y el transporte del cigoto a la cavidad uterina ocurran sin problema. Es importante resaltar que el daño de la mucosa tubárica no se puede restaurar con la cirugía.

\section{RESULTADOS DE LA CIRUGÍA TUBÁRICA}

Las adherencias pélvicas generalmente son secundarias a salpingitis, apendicitis previa, endometriosis pélvica, infección puerperal o cirugías ginecológicas previas. Los resultados después de la lisis de las adherencias dependen de la presencia concurrente de daño endosalpingueal y de la severidad de enfermedad extratubárica. Las adherencias pélvicas se pueden tratar por laparotomía -macroscópicamente o microscópicamente- o por laparoscopia usando disección roma o cortante, electro cauterio o láser.

\section{OVARIOLISIS Y SALPINGOLISIS}

Tulandi y colaboradores compararon dos grupos de pacientes con pocas diferencias en la extensión de las adherencias. A un grupo de 69 pacientes se les realizó adhesiolisis mediante técnica microquirúrgica, mientras a otro grupo de 78 pacientes no se les realizó ningún tratamiento; las tasas de embarazo después de uno y dos años de tratamiento fueron 32 y $45 \%$ respectivamente, en el grupo tratado, y de 11 y $16 \%$ en el grupo no tratado. Este estudio revela que la tasa de concepción después de salpingoovariolisis es tres veces más alta, sugiriendo que las adherencias juegan un papel en la infertilidad.

\section{MICROCIRUGÍA}

Se han reportado salpingoovariolisis por microcirugía utilizando tijeras, eletrocauterio y láser de $\mathrm{CO}_{2}$. Las tasas de embarazo varían entre 36.7 (12) y 75\% (13). El análisis de la literatura revela una tasa del $51.2 \%$ de embarazo promedio después de adhesiolisis por microcirugía, y una tasa del $47.6 \%$ de embarazo intrauterino. 
Fayez (13) y Donnez (14) observaron que dos tercios de los embarazos ocurrían durante el primer año de realizado el procedimiento. Tulandi y colaboradores (15) reportaron un intervalo promedio de 9.9 meses entre la intervención y la concepción.

El efecto de las diferentes técnicas se ha estudiado varias veces; Tulandi $y$ colaboradores (15) demostraron que no había ninguna diferencia en las tasas de embarazos después de salpingoovariolisis usando láser de $\mathrm{CO}_{2}$ o corriente eléctrica aplicada con electrodo de punta fina, aunque el intervalo entre la cirugía y la concepción es más corto con el uso del láser (3.2 meses). Watson y colaboradores (16) enfatizan el papel del experto en esta clase de cirugía. Ellos encontraron una tasa de embarazo mucho más baja (21\%) en dos centros hospitalarios no especializados que realizaban el procedimiento.

En la Tabla 1 se muestran los resultados de salpingoovariolisis por microcirugía.

\section{Laparoscopia}

La adhesiolisis por laparoscopia se puede realizar con tijeras (8-9), electro cauterio (17) o láser de $\mathrm{CO}_{2}(18)$.

En 1993, Gomel publicó un estudio retrospectivo de una serie de 92 pacientes en los cuales realizó salpingoovariolisis por laparoscopia con tijeras (8). El 62\% de los pacientes obtuvo por lo menos un embarazo intrauterino, y 54 pacientes (58.7\%) llevaron a término su embarazo. En cinco pacientes se presentó un embarazo ectópico (5.4\%). Resultados comparables fueron obtenidos por Fayez (9) utilizando tijeras para realizar el procedimiento por laparoscopia. Los autores puntualizan que la tasa de embarazo ectópico es un reflejo del daño del endosalpinx relacionado con el proceso de adherencia.

\section{Tabla 1}

Resultado de salpingoovariolisis después de microcirugía

\begin{tabular}{|lcccc|}
\hline Referencia & $\mathbf{n}$ & $\begin{array}{c}\text { Tasa de embarazos Intrauterino } \\
\text { (\%) }\end{array}$ & $\begin{array}{c}\text { Ectópico } \\
\text { (\%) }\end{array}$ \\
\hline Fayez (1982) & 8 & 75.0 & 100.0 & 0.0 \\
Frantzen & 49 & 40.8 & 36.7 & 4.1 \\
$(1982)$ & & & & \\
Donnez (1986) & 42 & 66.7 & 64.0 & 2.0 \\
Tulandi (1986) & 63 & 57.1 & 52.4 & 4.8 \\
Tulandi (1990) & 69 & 49.3 & 42.0 & 7.2 \\
Singhal (1991) & 78 & 46.2 & 41.0 & 5.1 \\
Total & 309 & 51.2 & 47.6 & 4.9 \\
\hline
\end{tabular}

Tabla 2

Resultado de salpingoovariolisis por laparoscopia

\begin{tabular}{|lccccc|}
\hline Referencia & $\mathbf{n}$ & $\begin{array}{c}\text { Tasa de } \\
\text { embarazos (\%) }\end{array}$ & \multicolumn{2}{c|}{ Intrauterino } & Ectópico Parto \\
& & 67.4 & 62.0 & 5.4 & 58.7 \\
\hline $\begin{array}{l}\text { Gomel } \\
\text { (1983) }\end{array}$ & 92 & & & & \\
Fayez (1983) & 50 & 60.0 & 56.0 & 4.0 & 46.0 \\
Donnez(1989) 186 & 58.0 & & & \\
Total & 328 & 61.0 & 59.8 & 4.9 & 54.2 \\
\hline
\end{tabular}


Donnez (18), utilizando láser de $\mathrm{CO}_{2}$ para realizar la adhesiolisis, obtuvo una tasa de embarazo del 58\%, sugiriendo que no hay diferencia entre el uso de las diferentes técnicas. En la Tabla 2 se muestran los resultados de salpingoovariolisis por laparoscopia.

Finalmente, los resultados de adhesiolisis por laparotomía frente a la laparoscopia no han sido establecidos en estudios prospectivos y controlados. $\mathrm{Al}$ respecto se puede concluir que la salpingoovariolisis por laparoscopia debe ser la primera elección de tratamiento quirúrgico para mujeres con adherencias peri anexales. Se puede esperar una tasa alta de embarazo intrauterino y una baja tasa de de embarazo ectópico, debido a que la disrupción de la anatomía y función tubárica es mínima en estas pacientes.

Pacientes con adherencias gruesas, vasculares y extensas tienen un pronóstico pobre de fertilidad después de cirugía (1921). Por lo tanto en presencia de adherencias anexales, la fertilización in vitro (FIV) se debe reservar para aquellas pacientes con enfermedad severa o quienes han fallado en concebir después de un año del tratamiento quirúrgico.

Entre los factores causantes de esta patología se encuentra con gran frecuencia la EPI, comprometiendo en la mayoría de los casos el endosalpinx (2, 21). Las tasas de embarazo de fimbrioplastia por microcirugía son comparables a las obtenidas después de adhesiolisis, con un promedio alrededor del $60 \%$ (Tabla 3 ).

Las tasas de embarazo de fimbrioplastia por laparoscopia están alrededor de un $35 \%$, con una tasa de embarazo ectópico entre el 9.7 y $14 \%(9,23)$.

Cuando se comparan estos resultados con la salpigoovariolisis se encuentra que son menos favorables, probablemente reflejan una afección más seria de la mucosa salpingeal, estos resultados se muestran en la Tabla 4.

\section{Salpingoneostomía}

La oclusión distal completa de la tuba con varios grados de formación de hidrosalpinx es secuela de una enfermedad pélvica inflamatoria severa. Generalmente se encuentra disfunción tubárica a causa del daño de la mucosa.

El objetivo de la cirugía es crear un nuevo ostium tubárico incidiendo la serosa y la pared tubárica. La eversión del endosalpinx se puede lograr por medio de suturas microquirúrgicas, electro cauterio o láser.

\section{Tabla 3}

Resultado fimbrioplastia por cirugia

\begin{tabular}{|lcccc|}
\hline \multicolumn{1}{|c}{ Referencia } & n & $\begin{array}{c}\text { Tasa de embarazos } \\
(\mathbf{\%})\end{array}$ & $\begin{array}{c}\text { Intrauterino } \\
(\mathbf{\%})\end{array}$ & $\begin{array}{c}\text { Ectópico } \\
(\%)\end{array}$ \\
\hline Fayez (1982) & 7 & 57.1 & 57.1 & 0.0 \\
Dotitez (1986) & 132 & 61.4 & 59.8 & 1.5 \\
Lavy (1987) & 134 & 59.7 & 53.7 & 5.9 \\
Total & 273 & 60.4 & 56.8 & 3.7 \\
\hline
\end{tabular}

Tabla 4

Resultado de fimbrioplastia por laparoscopia

\begin{tabular}{|lcccc|}
\hline \multicolumn{1}{|c}{ Referencia } & n & Tasa de embarazos (\%) & Intrauterino (\%) & Ectópico (\%) \\
\hline Gomel (1983) & 12 & & 50.0 & \\
Fayez (1983) & 14 & 35.0 & 21.0 & 14.0 \\
Dubuisson (1990) & 31 & 35.5 & 25.8 & 9.7 \\
Total & 57 & 35.0 & 30.0 & 11.0 \\
\hline
\end{tabular}


Gomel (24) es uno de los padres de la salpingoneostomía microquirúrgica; sus resultados, en una serie de 41 pacientes a los cuales se les realizó el procedimiento utilizando microdiatermia y microsuturas, arrojaron una tasa de embarazo del 29\% al año del procedimiento, con una tasa de niño en casa del $27 \%$. Debido a que todos los embarazos ectópicos y a que únicamente el $40 \%$ de los embarazos uterinos ocurrieron durante el primer año siguiente a la cirugía, Gomel concluyo que la restauración de la mucosa y la musculatura tubárica con restablecimiento de la permeabilidad y funcionalidad es dependiente del tiempo.

La revisión de la literatura revela una tasa de embarazo después de realizar la salpingostomía por microcirugía entre el 27 y $43 \%$, con una tasa de embarazo ectópico sorprendentemente alta (7.8\%). Los resultados de salpingostomía se describen en la Tabla 5.

Tabla 5

Resultado de salpingostomia por microcirugia

\begin{tabular}{|lccccc|}
\hline \multicolumn{1}{|c}{ Referencia } & n & Tasa de embarazos Intrauterino Ectópico Parto \\
& & $(\mathbf{0})$ & $\mathbf{( \% )}$ & $\mathbf{( \% )}$ & $\mathbf{( \% )}$ \\
\hline Gomel (1978) & 41 & 39.0 & 26.8 & 12.2 & 26.8 \\
Fayez (1982) & 20 & 40.0 & 35.0 & 05.0 & 35.0 \\
Tulandi (1985) & 67 & 26.9 & 22.4 & 04.5 & \\
Donnez (1986) & 83 & 38.5 & 31.3 & 07.2 & \\
Schlaff (1990) & 95 & 27.4 & 20.0 & 07.4 & \\
Singhal (1991) & 97 & 40.2 & 34.0 & 06.2 & 28.8 \\
Winston (1991) & 323 & 42.7 & 32.8 & 09.9 & 22.9 \\
Strandell (1995) & 109 & 35.8 & 22.9 & 12.8 & 18.3 \\
Total & 835 & 37.8 & 28.2 & 08.9 & 23.7 \\
\hline
\end{tabular}

Tabla 6

Resultado de salpingostomia por laparoscopia

\begin{tabular}{|lcccc|}
\hline \multicolumn{1}{|c}{ Referencia } & $\mathbf{n}$ & $\begin{array}{c}\text { Tasa de } \\
\text { embarazos (\%) }\end{array}$ & $\begin{array}{c}\text { Intrauterino } \\
\mathbf{( \% )}\end{array}$ & $\begin{array}{c}\text { Ectópico } \\
\mathbf{( \% )}\end{array}$ \\
\hline Fayez (1983) & 19 & 11.0 & 0.0 & 11.0 \\
Daniell (1984) & 21 & 24.0 & 19.0 & 5.0 \\
Donnez (1989) & 25 & 20.0 & & \\
Dubuisson & 34 & 32.4 & 29.4 & 2.9 \\
(1990) & & & & \\
Canis (1994) & 87 & 40.2 & 33.3 & 6.9 \\
Dubuisson(1994) 81 & 37.0 & 32.1 & 4.9 \\
Dlugi (1994) & 113 & 20.4 & 15.0 & 5.3 \\
Total & 380 & 29.2 & 24.2 & 5.0 \\
\hline
\end{tabular}


Cuando se comparan resultados de salpingostomía por microcirugía y macrocirugía se encuentra que las tasas de embarazo son sensiblemente iguales, $27 \mathrm{y}$ $26 \%$ respectivamente, siendo la tasa de embarazo ectópico mayor en la microcirugía que en macrocirugía, 7.8 y $4.3 \%$ respectivamente $(22)$.

El uso del láser de $\mathrm{CO}_{2}$ para salpingoneostomía no ofrece una ventaja clara sobre el electro cauterio en términos de aumentar la incidencia de embarazo o

Los factores que influyen en el éxito de la salpingostomía incluyen el diámetro del hidrosalpinx, la apariencia de la fimbria después de la eversión, la presencia de adherencias peri ováricas y el patrón de pliegues de la mucosa tubárica a la histerosalpingografía (28). Únicamente un $7 \%$ de las pacientes con

Cuando los estudios preliminares sugieren hallazgos de adherencias, fimbria aglutinada, mucosa aplanada, éstos se correlacionan con un pronóstico pobre de embarazo (30). Muchas de las evidencias parecen indicar que si el embarazo no ocurre dentro de un tiempo razonable, la probabilidad de un resultado exitoso es poca.

Audibert y colaboradores (31) estudiaron 226 pacientes con infertilidad tubárica distal que les fue corregida microquirúrgicamente o por laparoscopia; concluyeron que si el embarazo no había ocurrido entre los seis a doce meses después de la cirugía, recomendaban que estas pacientes entraran directamente al programa de FIV.

Con base en la evidencia publicada, a aquellas pacientes jóvenes con enfermedad tubárica mínima o moderada se les debe ofrecer cirugía reconstructiva, mientras aquéllas con enfermedad tubárica severa en quienes el beneficio potencial de una cirugía reconstructiva es limitado deberían ser tratadas primeramente con FIV, lo mismo que aquellas pacientes que no han concebido dentro del primer año de cirugía (32).

\section{OBSTRUCCIÓN TUBÁRICA PROXIMAL}

$\mathrm{La}$ anastomosis cornual se realiza en casos de obstrucción cornual. Los resultados de este procedimiento dependen reducir la incidencia de embarazo ectópico (25-26). La salpingoneostomía laparoscópica se puede realizar con tijeras, electrodo de aguja unipolar o láser de $\mathrm{CO}_{2}$ (27).

La tasa de embarazo en salpingostomía laparoscópica después de un intervalo de seis meses a dos años es alrededor del $29 \%$, comparable a la tasa obtenida después de un procedimiento microquirúrgico, con una tasa de embarazo ectópico del 5\% (Tabla $6)$.

histerosalpingografía preoperatoria que indique un pronostico pobre (p. ej. hidrosalpinx de un diámetro mayor de $2 \mathrm{~cm}$, ausencia de pliegues en la mucosa) se embarazan frente al $70 \%$ de aquéllas clasificadas en la categoría de buen pronóstico

(29).

de la etiología del bloqueo. Novy y colaboradores (33) demostraron canulación tubárica histeroscópica en el 50\% de casos de falsos positivos de obstrucción tubárica reportados por histerosalpingografía.

La anastomosis tubo cornual bilateral está asociada con tasas de embarazo intrauterino que varían de $50 \%$ (34) a $71 \%$ (35), con tasas de embarazo ectópico de 0 a $40 \%$ (36).

Los intentos de corregir múltiples sitios de obstrucción en la trompa de Falopio (enfermedad tubárica bipolar) han producido resultados muy pobres (37). La obstrucción tubárica múltiple es una indicación para FIV y no se debe realizar tratamiento quirúrgico.

\section{Reversión de la esterilización}

La esterilización tubárica sigue siendo uno de los métodos más comunes de contracepción en el mundo; afortunadamente la reanastomosis tubárica puede restaurar la fertilidad. El éxito de la reversión de la esterilización tubárica depende de varios factores: la longitud de cada trompa al terminar el procedimiento, el tipo del procedimiento para la esterilización, la localización de la anastomosis a lo largo de la tuba, la edad de la paciente y la presencia de otro tipo de patología coexistente.

Se ha reportado que la anastomosis ístmico-ístmico es la más exitosa en cuanto a tasas de embarazo (81\%), y que la 
longitud de las tubas en centímetros después del procedimiento, multiplicada por un factor de 10 da una aproximación de la tasa de embarazo a término (38). La esterilización con anillos de Silatic o ganchos produce poco daño a la tuba y por tanto los resultados de la reanastomosis son mejores que cuando ésta es realizada con electro cauterio; esta última técnica produce una mayor incidencia de embarazos tubáricos (39).

Cuando se compara la técnica microquirúrgica con la macroquirúrgica los resultados con la primera son definitivamente mejores cuando se realiza la anastomosis tubárica (62\% frente a $44 \%$ ) y la tasa de embarazo ectópico es menor (2.3\% frente a 9.2\%) (22). Para la paciente joven en la categoría de buen pronóstico, la anastomosis tubárica microquirúrgica ofrece excelentes resultados y la oportunidad de tener múltiples embarazos. Al contrario, la reversión de la esterilización tubárica es controversial en mujeres mayores de 40 años.

La tasa de embarazo reportada en 1992 por la Sociedad de Tecnología para la Reproducción Asistida (SART) en mujeres de 40 años y mayores sin compromiso del factor masculino fue del $7.2 \%$ (partos por ovocitos recuperados) (40). De este modo, cuando existe función ovárica normal, la decisión que se tome acerca del tratamiento debe hacerse después de un análisis cuidadoso de las posibilidades de éxito y riesgos de cada opción; de lo anterior podemos concluir que se debe realizar FIV en todas aquellas pacientes con baja probabilidad para una reanastomosis debido a una inadecuada longitud tubárica, obstrucciones cornuales por fibrosis extensa y fimbriectomía distal.

Se debe tener especial atención en el factor edad, pues con cada año que pase después de los 40 la fecundabilidad decrece drásticamente y la FIV puede ofrecer de forma inmediata una posibilidad de embarazo sin la morbilidad potencial de una cirugía abdominal.

\section{EMBARAZO ECTÓPICO Y CIRUGÍA TUBÁRICA RECONSTRUCTIVA}

La asociación de cirugía tubárica y embarazo tubárico es bien reconocida. El embarazo tubárico resulta de una demora en el tránsito del embrión a través de la trompa de Falopio.

El riesgo de embarazo ectópico después de una cirugía tubárica es variable y depende de la condición previa de la trompa, del tipo de procedimiento realizado, la técnica utilizada y la pericia del cirujano. Reparaciones quirúrgicas imperfectas pueden originar la formación de sacos de mucosa ciegos, lo cual puede ser causa de un atrapamiento del óvulo que predispone a la paciente a un embarazo ectópico. Igualmente, las adherencias peritubáricas, que a menudo suelen ser una secuela de cirugía, pueden restringir la motilidad tubárica y de esta forma interferir con la captación y el transporte del óvulo.

Pacientes con historia de embarazo ectópico tienen un riesgo mayor de recurrencia. El riesgo de embarazo ectópico recurrente después de salpinguectomía en una mujer que desea fertilidad es del 15.4\% y el de embarazo uterino de un 36.5\% (41).

En la actualidad son utilizados comúnmente procedimientos quirúrgicos que hacen todo lo posible por conservar la trompa de Falopio al momento de remover el embarazo ectópico. La elección del procedimiento en pacientes con embarazo ectópico, hemodinámicamente estables, que desean preservar su potencial reproductivo, es realizar una salpingostomía lineal mediante laparoscopia quirúrgica (42-44). Las tasas de embarazo ectópico recurrente no son diferentes de aquéllas a las que se les realizó salpinguectomía. Son de particular preocupación las mujeres que han tenido embarazos ectópicos múltiples. La alta posibilidad de repetir un embarazo ectópico, y las bajas tasas de embarazo intrauterino en este grupo, nos hacen sugerir con firmeza que el tratamiento de elección es FIV, previa salpinguectomía profiláctica (41-44).

\section{RESULTADOS EN REPRODUCCIÓN ASISTIDA}

\section{Tasas acumulativas de embarazo}

Para dar a las parejas alternativas aceptables de tratamiento, el cirujano ginecólogo debe estar familiarizado con las tasas de éxito de los diferentes procedimientos, y con las variables comprometidas en un estimativo real de las 
posibilidades de embarazo. Los datos reportados en 1992 por la Sociedad de Tecnología en Reproducción Asistida (SART) FIV-TE (embriones transferidos) (40) muestran que la tasa de partos por aspiración en 37.164 ciclos estimulados para FIV, GIFT y ZIFT fueron 16.8\%, 26.7 y $22.8 \%$ respectivamente; además, de 5.324 embriones crío-preservados y transferidos se obtuvo una tasa de partos del $11.5 \%$.

Las tasas de embarazo reportadas por el Registro Latinoamericano de Reproducción Asistida (44) muestran los siguientes resultados (Tabla 7).

El número total de procedimientos realizados por la Red durante 1999 incluye:

- Ciclos iniciados de FIV, GIFT y otros: 4.348 con tasas de embarazo clínico por aspiración de 27.0, 36.1 y $27.5 \%$ respectivamente.

- Ciclos aspirados de ICSI, ICSI + FIV, ICSI + GIF, Hatching Asistido: 8.298 con tasas de embarazos por aspiración de 26.5, 28.8, 33.0 y $26.8 \%$ respectivamente.

- Ciclos transferidos de crío preservación y ovodonación (OD): 2.236 con tasa de embarazo del $18.6 \%$ por trasferencia de embriones criopreservados, del $35.7 \%$ para óvulos donados frescos y $26.5 \%$ para óvulos congelados.

Del reporte de la Red Latinoamericana encontramos que las tasas acumulativas de embarazo clínico de parejas con factor tubárico (34.7\%) como causa de infertilidad tratadas con FIV es del $25.3 \%$ en 1.322 aspiraciones. Al relacionar las tasas de embarazo con la edad se encontró lo siguiente: pacientes menores de 35 años, 30.8\%; entre 35-39 años, 20.8\%; de 40 años o más, $12.8 \%$ (Tabla 8).

Es importante anotar que el estado de la enfermedad tubo ovárica o la historia de adherencias pélvicas o cirugía tubárica no parece tener un impacto significativo sobre la eficiencia del FIV. No obstante, se observa una disminución significativa en las tasas de embarazo en pacientes con factor tubárico como causa de infertilidad tratadas con FIV cuando se compararon los diferentes grupos de edad.

\section{El factor edad}

Uno de los factores pronósticos más importantes de la fertilidad humana es la edad de la mujer (45).
La disminución de la fertilidad de las parejas casadas con el avance de la edad ha sido documentada en forma repetida (46). Con seguridad se puede decir que un tercio de las mujeres que difieren el embarazo hasta mediados o finales de sus 30 años tienen problemas de fertilidad, y por lo menos la mitad de las mujeres por encima de los 40 años de edad experimentan problemas para concebir. Esta disminución en la fertilidad se inicia por lo menos 15 años antes de la menopausia; durante este periodo los ciclos menstruales son regulares, pero se observa un acortamiento estable del ciclo menstrual, debido a una fase folicular corta (47). Con el acortamiento de los ciclos al final de los 30 años se produce un incremento sutil pero real de la FSH, y una disminución de los niveles de inhibina (48). Estos cambios se asocian a menudo a un crecimiento folicular acelerado, aunque al mismo tiempo se desarrollan pocos folículos por ciclo; finalmente ocurre la menopausia porque el abastecimiento de folículos se agota.

La disminución de la fecundidad femenina con la edad se ha observado en FIV y en otras técnicas de reproducción asistida (49-50). Muchos programas de reproducción asistida han establecido una edad límite basados en el pronóstico marcadamente pobre para mujeres por encima de los 40 años a causa de las bajas tasas de embarazo y a un incremento en el riesgo de aborto espontáneo (51).

A diferencia de los hombres, las mujeres están dotadas de un número finito no reemplazable de células germinales que alcanzan el pico máximo a la mitad de la gestación (52).

El número absoluto y la capacidad funcional de los folículos y células germinales comprende lo que podemos llamar la reserva ovárica, lo cual afecta su respuesta a la estimulación y su posibilidad de éxito. Se han descrito varias pruebas para evaluar la reserva ovárica en forma indirecta entre éstas se incluyen:

Pruebas estáticas, que se caracterizan por la determinación en situación basal (día tres del ciclo) de una determinada sustancia (52-60): FSH, estradiol sérico $\left(E_{2}\right)$ e inhibina B. Pruebas dinámicas, que se caracterizan por la determinación de FSH y $\mathrm{E}_{2}$ antes $\mathrm{y}$ después de someter al eje 
hipotálamo-hipófisis a un estímulo específico (56-61): El Efort (Exogenous FSH Ovarian Reserve Test) y la prueba del clomifeno. Estas pruebas se deben realizar en todas las mujeres mayores de 35 años, ya que a partir de esta edad existe la mayor proporción de pacientes con baja respuesta a la estimulación ovárica; no obstante, no todas presentaran una baja respuesta a la hiperestimulación ovárica controlada. Por otro lado, la calidad ovocitaria desciende con la edad (62).

Tabla 7

Ciclos do tratariento y embarazo clinico segin tipo de procedirtento (1999)

\begin{tabular}{|c|c|c|c|c|c|c|c|c|c|c|c|c|}
\hline \multicolumn{13}{|c|}{ PROCEDIMLENTOS } \\
\hline & & & & \multicolumn{7}{|c|}{ MICROMANIPULACION } & \multirow{2}{*}{\multicolumn{2}{|c|}{$\begin{array}{c}\text { OVO } \\
\text { DONACION }\end{array}$}} \\
\hline & & & & \multirow[t]{2}{*}{ ICSI } & \multirow{2}{*}{$\begin{array}{l}\text { ICSI } \\
+ \text { FIV }\end{array}$} & \multirow{2}{*}{$\begin{array}{l}\text { SOFT/ } \\
\text { TOMI }\end{array}$} & $\begin{array}{l}\text { HATCHING } \\
\text { ASISTIDO }\end{array}$ & \multicolumn{3}{|c|}{$\begin{array}{l}\text { CRIO PRESER- } \\
\text { VACION }\end{array}$} & & \\
\hline & FIV & GIFT & OTROS & & & & FIV & ICSI & FIV & ICSI & $\begin{array}{l}\text { FRES- } \\
\text { COS }\end{array}$ & $\begin{array}{l}\text { CONGE. } \\
\text { LADOS }\end{array}$ \\
\hline $\begin{array}{l}\text { CICLOS } \\
\text { INICIADOS }\end{array}$ & & & & 6623 & $35 !$ & 105 & 85 & 1134 & & & & \\
\hline $\begin{array}{l}\text { CICLOS } \\
\text { DESCONTI. } \\
\text { NUADOS }\end{array}$ & 526 & 12 & 1 & 524 & 18 & 2 & 0 & 46 & & & & \\
\hline $\begin{array}{l}\text { ASPIRA. } \\
\text { CIONES (A) }\end{array}$ & 3686 & 72 & 51 & 6099 & 336 & 103 & 85 & 1088 & & & & \\
\hline $\begin{array}{l}\text { TRANSFE } \\
\text { RENCIAS (B) }\end{array}$ & 3166 & 71 & 42 & 5568 & 319 & 102 & 85 & 1025 & 386 & 592 & 1150 & 98 \\
\hline $\begin{array}{l}\text { EMBARAZOS } \\
\text { CLINICOS (C) }\end{array}$ & 996 & 26 & 14 & 1615 & 96 & 34 & 24 & 275 & 73 & 108 & 411 & 26 \\
\hline $\begin{array}{l}\text { PARTOS >1 } \\
\text { RN(S) (D) }\end{array}$ & 754 & 20 & 13 & 1211 & 71 & 30 & 15 & 2007 & 51 & 78 & 304 & 16 \\
\hline $\begin{array}{l}\% \text { CICLOS } \\
\text { DESCONTI. } \\
\text { NUADOS }\end{array}$ & 12.5 & 143 & 1.9 & 79 & 3 & 1.9 & - & 4 & & & & \\
\hline \multicolumn{13}{|c|}{ \% DE TRAWSFERENCIAS POR: } \\
\hline ASPIRACION & 85.9 & 98.6 & 82.4 & 91.3 & 95.1 & 99 & 100 & & & & & \\
\hline \multicolumn{13}{|c|}{ \% DEEMBARAZO CLINIOO POR: } \\
\hline ASPIRACION & 27 & 36.1 & 275 & 26.5 & 28.8 & 33 & 28.2 & & & & & \\
\hline $\begin{array}{l}\text { TRANSFE } \\
\text { RENCIA }\end{array}$ & 31.5 & 36.6 & 33.3 & 29 & 30.3 & 33.3 & 28.2 & & 189 & 18.2 & 35.7 & 26.5 \\
\hline \multicolumn{13}{|c|}{$\%$ DE PARTO $(\$)$ CON $>1$ RN(S) POR: } \\
\hline ASPIRACION & 20.5 & 278 & 255 & 199 & 20.3 & 29.1 & 17.6 & & & & & \\
\hline $\begin{array}{l}\text { TRANSFE } \\
\text { RENCIA }\end{array}$ & 23.8 & 282 & 31 & 21.7 & 21.4 & 29.4 & 17.6 & & 132 & 13.2 & 26.4 & 16.3 \\
\hline $\begin{array}{l}\text { EMBARAZO } \\
\text { CLINICO }\end{array}$ & 75.7 & 769 & 929 & 75 & 70.7 & 88.2 & 62.5 & & 699 & 722 & 74 & 61.5 \\
\hline
\end{tabular}

A. ASPIRACIONES: se refiere a aspiraciones foliculares transvaginales y laparoscopias, independientemente de la te cuperación de ovocitos.

B. TRANSFERENCIAS: se refiere a la transferencia al útero y/o trompa de Falopio de una o más conceptus (embriones) y/o gametos. C. EMBARAZOS CLINICOS: se refiere a gestaciones intrauterinas documentadas ecogrfic amente con il menos un saco gestacional. DPARTOS se refiere a los recién nacidos después de completadas las 20 semanas de gestación.

El número total de procedirtientos realizados durante 1999 incluye:

Ciclos iniciados de FIV, GIFT, otros y mictomanipulación $=12.652$ ciclos.

Ciclos transferidos de criopreservación y ovodonación $(\mathrm{OD})=2226$ ciclos. 
Tabla 8

Tasa de embarazo clínico según categoria diagnóstica

\begin{tabular}{|lllllllllll|}
\hline \multicolumn{2}{|c|}{ EDAD (años) TUBARIA } & \multicolumn{3}{c|}{ OTRAS } & \multicolumn{4}{c|}{ MASCULINAS MULTPLE INEXPLICADA } \\
& ASP & $\%$ & ASP & $\%$ & ASP & \% & ASP & \% & ASP & $\%$ \\
\hline$<35$ & 701 & 30.8 & 504 & 32.5 & 238 & 30.7 & 367 & 31.3 & 191 & 31.4 \\
$35-39$ & 496 & 20.8 & 323 & 28.2 & 153 & 30.1 & 267 & 21.3 & 134 & 36.6 \\
$>40$ & 125 & 12.8 & 123 & 16.3 & 47 & 10.6 & 86 & 11.6 & 54 & 16.7 \\
TOTAL & 1322 & 25.3 & 950 & 28.9 & 438 & 28.3 & 720 & 25.3 & 379 & 31.1 \\
\hline
\end{tabular}

Se define como baja respondedora a una mujer con ciclos ovulatorios espontáneos completamente normales (se excluyen todas alteraciones menstruales asociadas a un desarrollo folicular anormal como el síndrome de ovario poliquístico y la premenopausia), que responde a los protocolos habituales de inducción de ovulación con menos de tres a seis folículos en, al menos, dos ciclos consecutivos de estimulación (un sólo ciclo de baja respuesta puede obedecer a motivos ajenos al funcionamiento del ovario). Un estradiol $\left(E_{2}\right)$ menor de $500 \mathrm{pg} / \mathrm{ml}$ tras finalizar un ciclo de hiperestimulación ovárica controlada, podría indicar un ciclo de baja respuesta, pero en la actualidad es un parámetro poco útil en la clínica.

\section{Determinación de la FSH sérica}

La FSH sérica tiene un valor predictivo de los resultados de éxito de la FIV, de manera que mujeres con FSH basal mayor de $20 \mathrm{mUl} / \mathrm{ml}$ el día tres del ciclo tiene mal pronóstico (54). Incluso se ha comprobado que la FSH se correlaciona mejor que la edad con la probabilidad de éxito de la FIV (55).

\section{El $E_{2}$}

La elevación del $\mathrm{E}_{2}$ en el día tres del ciclo menstrual es indicador de un pronóstico pobre y se debe al aumento en la secreción de la FSH en la fase lútea del ciclo anterior, lo que produce una foliculogénesis acelerada $y$, por consiguiente, niveles de $\mathrm{E}_{2}$ mayores de 30 $\mathrm{pg} / \mathrm{ml}$.

Debido a la variabilidad entre laboratorios, se recomienda la determinación de estos valores en dos

ocasiones diferentes antes de tomar una decisión clínica.

\section{La inhibina $B$}

La inhibina B una glucoproteína secretada directamente por las células de la granulosa del folículo, sus niveles son variables durante el ciclo menstrual, y presenta niveles más bajos en mujeres mayores de 35 años sometidas a hiperestimulación ovárica controlada; por esta razón es indicador sensible de la vitalidad de los folículos en crecimiento (59-60).

Seifer y colaboradores demostraron que las mujeres con niveles séricos basales de inhibina $B$ menores de 45 $\mathrm{pg} / \mathrm{ml}$ el día tres tenían malas respuestas a la hiperestimulación ovárica controlada que aquéllas con niveles de inhibina $\mathrm{B}$ mayores o iguales a $45 \mathrm{pg} / \mathrm{ml}$.

\section{El Efort}

Consiste en medir los niveles basales de FSH y $E_{2}$ administrando a continuación 300 UI de FSH el día tres del ciclo, midiendo de nuevo FSH y $\mathrm{E}_{2}$ sérico a las 24 horas. Si la FSH es mayor de $11 \mathrm{mUI} / \mathrm{ml}$ y/o el incremento de $\mathrm{E}_{2}$ es menor de $30 \mathrm{pg} / \mathrm{ml}$ se habla de un Efort patológico; pero si la FSH es menor de $11 \mathrm{mUI} / \mathrm{ml} \mathrm{y} / \mathrm{o}$ el incremento de $E_{2}$ es mayor de $30 \mathrm{pg} / \mathrm{ml}$, se habla de un Efort normal (56). Esta prueba tiene un alto valor predictivo: el $90 \%$ de las mujeres con un Efort normal mostraron una respuesta óptima a la estimulación ovárica controlada; por el contrario, con un Efort patológico, el $81 \%$ de las pacientes presentaron respuestas bajas (56). 


\section{Prueba de clomifeno}

Ésta ha sido diseñada para explicar situaciones clínicas confusas, en las que mujeres con respuesta baja a la hiperestimulación ovárica controlada presentan niveles basales de FSH normales o ligeramente elevados (menores de 20 $\mathrm{mUI} / \mathrm{ml})(53,57)$.

La prueba consiste en medir la FSH sérica el día tres del ciclo, y estimular a la mujer con $100 \mathrm{mg} /$ diarios de citrato de clomifeno durante los días cinco al nueve del ciclo, y determinar nuevamente la FSH el día 11 del ciclo. Si la sumatoria de las dos FSH es mayor de $26 \mathrm{mUI} / \mathrm{ml}$ se debe considerar que la paciente está en falla ovárica.

La relación entre concentraciones elevadas de FSH y los resultados de la FIV es sorprendente. El efecto se observa en el patrón de la respuesta ovárica a la estimulación, tasas de embarazo y eficiencia en la implantación.

Los resultados de tasas de embarazo por aspiración caen de un $21.1 \%$ en mujeres con FSH menores de10 $\mathrm{mU} / \mathrm{ml}$ el día tres del ciclo a $8.3 \%$ en mujeres con niveles basales de FSH por encima de $20 \mathrm{mUI} / \mathrm{ml}$ (64).

Parece que el mayor obstáculo para la concepción natural en la mujer mayor está relacionado con una disminución en la implantación del embrión: la tasa de implantación por embrión diminuye de $29 \%$ en mujeres menores de 34 años, a $21.8 \%$ en mujeres de 34 años, con una disminución lineal hasta $2.2 \%$ por embrión transferido a los 44 años (64).

En las mujeres agonadales que reciben embriones de donantes jóvenes su tasa de embarazo es de la más alta observada en reproducción asistida, sugiriendo que la disminución en la tasa de implantación con la edad avanzada está claramente relacionada con la pobre calidad del oocito (65). Hay gran cantidad de evidencia que demuestra que embriones obtenidos de mujeres de 40 años a los cuales se les ha realizado diagnóstico genético preimplantación presentan con gran frecuencia aneuploidias (66). Estos hallazgos son consistentes con las observaciones realizadas en pacientes de
FIV, quienes presentan alta tasa de abortos espontáneos con la edad avanzada (67).

Debido a la rápida disminución de la fertilidad en mujeres sobre los 40 años de edad, a éstas se les debe realizar una evaluación rápida. La determinación de FSH y $E_{2}$ el día tres del ciclo es de gran ayuda cuando se trata de aconsejar a la paciente acerca de reales posibilidades de embarazo o de dirigirla con niveles altos de gonadotropinas a recibir óvulos de una donante.

La reparación quirúrgica o laparoscópica de la enfermedad tubárica se debe cuestionar seriamente en aquellas mujeres que están al final de la década de los 30, en quienes (aún cuando los niveles de gonadotropinas estén normales el día tres a la evaluación inicial) la reserva ovárica puede declinar, disminuyendo la posibilidad de un embarazo exitoso. En este grupo particular de pacientes creemos que los esfuerzos se deben dirigir a un método de reproducción asistida que podría proporcionar una mayor posibilidad de éxito en un intervalo de tiempo corto.

\section{DISCUSIÓN}

La infertilidad tubárica debida a causas infecciosas va en aumento, esto ha causado un creciente interés en la cirugía reconstructiva. En la década pasada se observó un cambio en el tratamiento quirúrgico de los desórdenes tuboperitoneales. La laparoscopia quirúrgica y las técnicas microquirúrgicas prácticamente han reemplazado a la laparotomía. Como quiera que sea, la evolución de la microcirugía a la laparoscopia tomó lugar sin estudios prospectivos randomizados. El papel de las técnicas de reproducción asistida en la infertilidad tubárica no ha sido aún determinado de manera prospectiva controlada.

Toda pareja infértil, con factor tubo peritoneal comprometido, debe ser estudiada en forma completa, esto incluye espermograma, evaluación de los niveles basales de FSH el día tres del ciclo, histerosalpingografía y laparoscopia 
diagnóstica. Estos estudios son decisivos programa de cirugía tubárica reconstructiva o al programa de técnicas de reproducción asistida.

Se ha demostrado el papel de la adhesiolisis en favorecer la fertilidad. Tulandi y colaboradores (11) mostraron que la salpingoovariolisis favorece la posibilidad de embarazo en un factor de tres. No se encontraron diferencias significativas en los resultados después de realizar la salpingoovariolisis por microcirugía o laparoscopia. Las tasas acumulativas de embarazo después de dos años de seguimiento están entre 50 y $60 \%$ (Fig. 1).

Tasas de embarazo por técnica microquirúrgica y laparoscopia $\mathrm{P}<0.05$ en fimbrioplastia

Las tasas de embarazo intrauterino son ligeramente más altas con el manejo laparoscópico, aunque esto no es estadísticamente significativo (Fig. 2).

No se encontraron diferencias en las tasas de embarazo ectópico (Fig. 3).

Si las adherencias son el único factor de infertilidad demostrable, la salpingoovariolisis laparoscópica debe ser el tratamiento de elección; no obstante los resultados están relacionados con la extensión de las adherencias. Hulka (21) demostró que pacientes a las que se les realizó salpingoovariolisis microquirúrgica de adherencias laxas (avasculares) alcanzaron una tasa de embarazo del $47 \%$ frente a $0 \%$ en pacientes con adherencias extensas (densas y vasculares), por tanto estas últimas pacientes deben ir directamente al programa de reproducción asistida.

Cuando existe obstrucción tubárica distal parcial, asociada con aglutinación de la fimbria o fimosis, el procedimiento a realizar es una fimbrioplastia.

En nuestra revisión encontramos un incremento estadístico de embarazo en favor de la fimbrioplastia por microcirugía (Fig. 1).

Los resultados en términos de embarazo intrauterino después de salpingolisis y fimbrioplastia por microcirugía son similares (Fig. 2).

El embarazo ectópico ocurre con una frecuencia tres veces mayor en grupos de fimbrioplastia por laparoscopia (Fig. 3). La para determinar si la pareja se refiere a razón para esta diferencia no es clara; se podría esperar una tasa grande de embarazo ectópico en los dos grupos por el compromiso endosalpingueal presente en ambos, pero posiblemente la restauración anatómica es mucho más precisa en la microcirugía que en la laparoscopia.

Cuando un hidrosalpinx está presente se debe realizar una salpingoneostomía. Usualmente se encuentra un compromiso severo de la mucosa tubárica, resultando en tasas bajas en embarazo. No se encontraron diferencias en los resultados (tasa de embarazo uterino y ectópico) con el manejo realizado por microcirugía o laparoscopia. Estos resultados dependen del estado anatómico y la integridad funcional de las trompas de Falopio. Muchas clasificaciones toman estos dos factores en cuenta.

Mage y colaboradores (67) clasifican los pacientes de acuerdo con la extensión de las adherencias perianexales y al estado de la tuba. Esta clasificación tiene fundamento en el tipo y grado de compromiso de las adherencias ováricas. La apariencia tubárica es evaluada con base en el aspecto de los pliegues de la mucosa a la histerosalpingografía y el aspecto de la pared salpingueal (puntajes de I a IV, el I es mejor). La clasificación de la American Fertility Society (1988) se basa en la mucosa tubárica, paredes de la tuba, tamaño del hidrosalpinx, tipo de adherencias y área comprometida.

En una serie de 81 pacientes a los cuales se les realizó salpingoneostomía por laparoscopia, Dubuisson y colaboradores (68) encontraron una tasa de embarazo del $60 \%$ en el grupo I y $0 \%$ en el grupo IV de acuerdo con el puntaje tubárico asignado por Mage y colaboradores (67). Estos autores recomiendan realizar salpingoneostomía en pacientes con daño tubárico mínimo y limitado, mientras que pacientes con daño tubárico severo se les debe aconsejar FIV.

Por otra parte, diversos autores puntualizan que similarmente a la salpingoovariolisis, la severidad de la patología tubárica preexistente y la extensión de las adherencias tubáricas juegan un papel que afecta las tasas de éxito. 

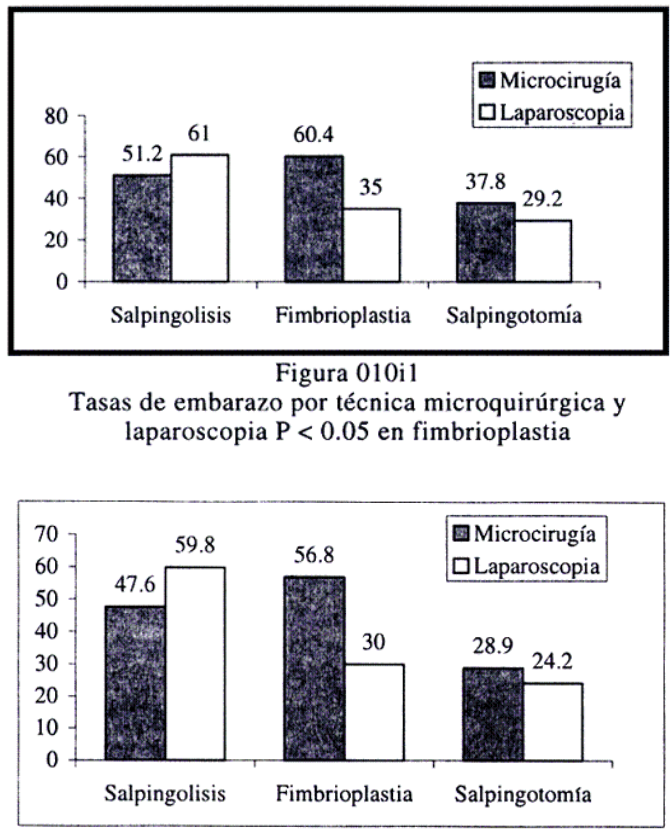

Figura 010i2

Tasas de embarazo intrauterino por técnica microquirúrgica y laparoscopia $\mathrm{P}>0.055$ en tres grupos

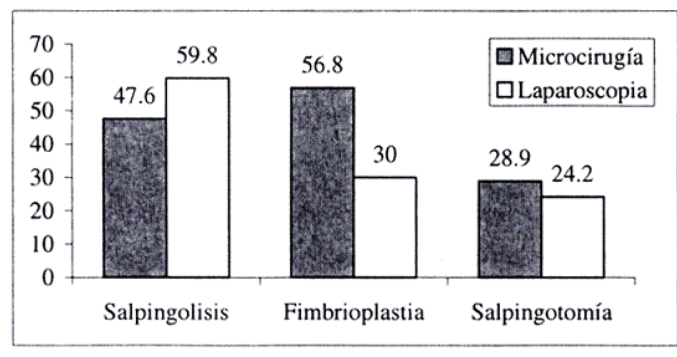

Figura $010 \mathrm{i} 3$

Tasas de embarazo ectópico por microcirugía y laparoscopia

El diámetro del hidrosalpinx es también un factor pronóstico importante. Singhal y colaboradores (69) encontraron tasas de nacimiento significativamente bajas en pacientes con hidrosalpinx mayores de 20 mm de diámetro comparado con aquéllos de diámetro menor de $20 \mathrm{~mm}$; de estos resultados se concluyó que este factor negativo es una consecuencia directa de una disminución de las células ciliadas del lumen tubárico distal.

El FIV con transferencia de embriones (TE) es una amplia alternativa disponible a la cirugía tubárica reconstructiva.

La pregunta que persiste es ¿̇a cuáles parejas se les debe ofrecer cirugía de infertilidad o técnicas de reproducción asistida?

Las tasas de embarazo clínico después de FIV-TE en pacientes con factor tubárico varía entre 30.8 y $12.8 \%$, dependiendo de la edad de la paciente, siendo las mejores tasas en mujeres menores de 35 años y bajas en mujeres de 40 años y más (45).

Benadiva y colaboradores ${ }^{2}$ estudiaron en forma retrospectiva la tasa de embarazo acumulativo en parejas con factor tubárico como causa de infertilidad; la tasa de parto total en pacientes con factor tubárico como causa exclusiva de infertilidad por transferencia fue de $30.1 \%$ en 491 pacientes (673 aspiraciones). Ellos demostraron que otros diagnósticos de infertilidad secundarios (anovulación, endometriosis, factor masculino, inmunológico) no afectan las tasas de embarazo (26\% en 371 aspiraciones) en forma significativa después de FIV-TE.

La tasa de embarazo acumulativa después de cuatro ciclos fue mayor del $70 \%$ en ambos grupos. Se observó una tasa de aborto del 2.4\%.

Se debe tener especial cuidado en pacientes con grandes hidrosalpinx. Los resultados de FIV- ET se ven afectados negativamente por el flujo retrógrado del líquido del hidrosalpinx a la cavidad uterina. La implantación se puede deteriorar por el efecto tóxico sobre el endometrio (70).

Si la cirugía reconstructiva es de mal pronóstico, se debe realizar salpinguectomía o ligadura en estas pacientes antes de iniciar técnicas de reproducción asistida.

Cuando consideramos las tasas acumulativas de embarazo después de cuatro ciclos de técnicas de reproducción asistida o salpingoovariolisis, fimbrioplastia o salpingoneostomía (Fig. 1), la primera da mejores resultados en cada uno de los frentes; sin embargo, podría ser un error pronosticar que la cirugía reconstructiva vaya a ser historia pronto. En primer lugar, si la cirugía es exitosa ésta ofrece la posibilidad de concebir en múltiples ciclos y lograr múltiples embarazos en forma consecutiva. El riesgo de embarazo múltiple es bajo después de tratamiento quirúrgico.

El factor económico también puede pesar al momento de tomar la decisión; en Colombia el costo de un tratamiento de reproducción asistida (FIV-TE) está entre $\$ 7.000 .000$ y $\$ 9.000 .000$, y una cirugía reconstructiva está en el promedio de 
\$4.000.000; el dilema está en seleccionar la paciente que tiene buen pronóstico quirúrgico.

El objetivo principal es determinar la técnica más eficaz con las mínimas complicaciones para cada pareja a fin de obtener un embarazo en el tiempo mínimo posible. La edad de la paciente y los niveles basales de la FSH juegan un papel importante en la decisión.

Los resultados anteriores sugieren que las mujeres jóvenes, con disturbio tuboperitoneal leve o moderado, son candidatas primarias para cirugía tubárica reconstructiva, mientras mujeres de edad mayor con enfermedad severa o pelvis congelada deben ser dirigidas inmediatamente al programa de reproducción asistida.

Resultados comparables se han obtenido en salpingoovariolisis y salpingoneostomías realizadas por laparoscopia o microcirugía, haciendo que el manejo laparoscópico sea el preferible; en el caso de ser necesaria una fimbrioplastia se debe considerar preferentemente realizarla por laparotomía microquirúrgica.

Las anteriores conclusiones se fundamentan en datos retrospectivos. No hay estudios randomizados sobre una base prospectiva, que comparen las tasas de embarazo después de una microcirugía o una laparoscopia quirúrgica y reproducción asistida en parejas con obstrucción tubárica demostrada y espermograma normal.

\section{REFERENCIAS BIBLIOGRÁFICAS}

1. Lilford RJ, Watson AJ. Has in vitro fertilization made salpingostomy obsolete? Br J Obstet Gynecol 1990; 97: 557-560.

2. Benadiva CA, Kligman I, Davis O, Rosenwaks Z. In vitro fertilization versus tubal surgery: is pelvic reconstructive surgery obsolete? Fertil Steril 1995; 64: 1051-1061.

3. Speroff L, Glass RM, Kase NG. Female infertility. In: Clinical gynaecologic endocrinology and infertility, $5^{\text {th }} \mathrm{Ed}$. Williams and Wilkins. Baltimore 1994; 809-839.

4. Trimbos-Kemper T, Trimbos B, Van Hall E. Etiological factors in tubal infertility. Fertil Steril 1982; 37: 384-388.

5. Westron L. Incidence, prevalence and trends of acute pelvic inflammatory disease and its consequences in industrialized countries. Am J Obstet Gynecol 1980; 138: 880-892.

6. Urman B, Gomel V, McComb P, Lee N. Midtubal occlusion: aetiology, management and outcome. Fertil Steril 1992; 57: 747-750

7. Sulak PJ, Letterie GS, Coddington CC, Hayslip CC, Woodward JE, Klein TA. Histology of proximal tubal occlusion. Fertil Steril 1987; 48: 437-440.

8. Gomel V. Salpingoovariolisis by laparoscopy in infertility. Fertil Steril 1983; 40: 607-611.
9. Fayez JA. An assessment of the role of operative laparoscopy in tuboplastia. Fertil Steril 1983; 39: 476-479. 10. Bowman MC, Cook ID. Comparison of fallopian tube intraluminal pathology as assessed by salpingoscopy with pelvic adhesions. Fertil Steril 1994; 61: 464-469.

11. Tulandi T, Collins JA, Burrows E, Jarrel JF, Mc Innes RA, Wrixon W, Simpson CW. Treatment dependent and treatment independent pregnancy among women with periadnexal adhesions. Am J Obstet Gynecol 1990, 162: 354-357.

12. Frantzen C, Schlosser HW. Microsurgery and post infectious tubal infertility. Fertil Steril 1982; 38: 397-402.

13. Fayez JA, Suliman SO. Infertility surgery of the oviduct: comparison between macrosurgery and microsurgery. Fertil Steril 1982; 37: 73-78.

14. Donnez J, Casanas-Roux F. Prognostic factors of fimbrial microsurgery. Fertil Steril 1986; 46: 200-204.

15. Tulandi T. Salpingoovarilysis: a comparison between laser surgery and electrosurgery. Fertil Steril 1986; 45: 489491.

16. Watson AJS, Gupta JK, O' Donovan P, Dalton ME, Lilford RJ. The results of tubal surgery in the treatment of infertility in two non-specialist hospital. $\mathrm{Br} \mathrm{J}$ Obstet Gynecol 1990; 97: 561-568.

17. Marama R, Rizzi M, Muzii L, Catalano GF, Carvana P, Mancuso S. Correlation between the American Fertility Society Classifications of adhnexal adhesions and distal tubal occlusion, salpingoscopy and reproductive outcome in tubal surgery. Fertil Steril 1995; 64: 924-929.

18. Donnez J, Nisolle $\mathrm{M}$, Casanas-Roux F. $\mathrm{CO}_{2}$ laser laparoscopy in infertility women with tubal occlusion. J Gynecol Surg 1989; 5: 47-53.

19. Siegler AM, Kontopoulos V. An analysis of macrosurgical and microsurgical techniques in the management of the tuboperitoneal factor in infertility. 1979; 32: $377-382$

20. Caspi E, Halperin Y. Surgical management of periadnexal adhesions. Int J Fertil 1981; 26: 49-54.

21. Hulka JF. Adnexal adhesions: a prognostic staging and classification system based of five year survey of fertility surgery results at Chapel Hill, North Carolina. Am J Obstet Gynecol 1982; 144: 141-148.

22. Lavy G, Diamond MP, De Cherney AH. Ectopic pregnancy: its relationship to tubal reconstructive surgery.

23. Dubuisson JB, Bouquet de Jolienere J, Aubriot FX, Darai E, Foulot H, Mandelbrot L. Terminal tuboplasties by laparoscopy: 65 consecutives cases. Fertil Steril 1990; 54: 401-403.

24. Gomel V. Salpingostomy by microsurgery. Fertil Steril 1978; 29: 380-387.

25. Tulandi T, Farag R, Mcinnes RA. Reconstructive surgery for hidrosalpinx and without the carbon dioxide laser. Fertile Steril 1984; 42: 839-845.

26. Mage G, Bruhat MA. Pregnancy following salpingostomy: comparison between $\mathrm{CO}_{2}$ laser and electrosurgery procedures. Fertil Steril 1983; 40: 472-478.

27. Tulandi T, Bugnah M. Operative laparoscopy: surgical modalities. Fertil Steril 1995; 63: 237-245.

28. Rock JA, Katayana KP, Martin EJ. Factors influencing the success of salpingostomy techniques for distal fimbrial obstruction. Obstec Gynecol 1978; 52: 591-596.

29. Young PE, Egan JE, Barlow J. Reconstructive surgery for infertility at the Boston Hospital for women. Am J Obstet Gynecol 1970; 108: 1092-1098.

30. Debruyne F, Puttemans P, Boeckx W. The clinical value of salpingoscopy in tubal infertility. Fertil Steril 1989; 51 : 339-346.

31. Audibert F, Hedon B, Arnal F. Therapeutic strategies in tubal infertility with distal pathology. Human Reprd 1991; 6: 1439-1445.

32. Marana R, Quagliarello J. Distal tubal occlusion: microsurgery versus in vitro fertilization a review. Int. J Fertil 1988; 33: 107-114.

33. Novy MJ, Thurmond AS, Patton P. Diagnosis of cornual obstruction by transcervical fallopian tube cannulation. Fertil Steril 1986; 46: 21-27. 
34. Kerin JF, Surrey ES, Williams DB. Falloscopic observations of endotubal isthmic plugs as a causal of reversible obstruction and their histological characterization. J Lparoscopic Surg 1991; 1:103-109.

35. Gomel V. Tubal reanastomosis by microsurgery. Fertil Steril 1977; 28: 59-65.

36. Lavy G, Diamond MP, DeCherney AH. Pregnancy following tubocornual anastomosis. Fertil Steril 1986; 46: 21-27.

37. Patton PE, William TJ, Coulan CB. Results of microsurgical reconstruction in patients with combined proximal and distal tubal occlusion: double obstruction. Fertil Steril 1987; 48: 670-677.

38. Henderson SR. The reversibility of female sterilization with the use of microsurgery: a report on 102 patients with more than one year of follow-up. Am J Obstet Gynecol 1984; 149: 57-64.

39. Rock JA, Bergquist CA, Zacur HA. Tubal anastomosis following unipolar cautery. Fertil Steril 1982; 37: 613-620. 40. Society For Assisted Reproductive Technology, The American Fertility Society: Assisted Reproductive Technology in the United States and Canada: 1993 results generates from The American Fertility Society /Society for Assisted Reproductive Technology Registry. Fertil Steril 1994; 62: 1121-1131.

41. Olsner G, Tarlatzis BC, Radical surgery for extrauterine pregnancy. In: Ectopic Pregnancy, De Cherney AH, editorial. Rockville, MD. Aspen Publishers. 1986: 127-132. 42. Bruhat MA, Manhes H, Mage G. Treatment of ectopic pregnancy by means of laparoscopy. Fertil Steril 1980; 33: 411-416.

43. Saavedra JS, Navarro NH, Díaz C. Embarazo ectópico: manejo ambulatorio. Rev Colomb Obstet Ginecol 2001; 52: 79-85.

44. Registro Latinoamericano de Reproducción Asistida. Zegers F, Gadalmes V, Balmaceda J. Ciclos de tratamiento y embarazo clínico según tipo de procedimiento 1999.

45. Tietze C. Reproductive span and rate reproduction among Hutterite women. Fertil Steril 1957; 8: 59-64.

46. Menken J, Trussell J, Larsen U. Age and infertility. Science 1986; 233: 1389-1395.

47. Sherman BM, Korenman SG. Hormonal Characteristics of human menstrual cycle troughout reproductive life. J Clin Invest 1975; 55: 699-705.

48. Lenton EA, Dekretser DM, Woodward AJ. Inhibin concentrations throughout the menstrual cycle of normal, infertile and older women compared with those during spontaneous conceptions cycles. J Clin Endocrinol Metab 1991; 73: 1180-1186.

49. Sharma V, Riddle A, Mason BA. An analysis of a clinical pregnancy in an ultrasound based ambulatory in vitro fertilization program. Fertil Steril 1988; 49: 468-474.

50. Piette C, De Mouzon J, Buchelot A. In vitro fertilization: influence of women's age on pregnancy rates. Human Reprod 1990; 5: 56-62.

51. Romeu A, Muasher SJ, Acosta AA. Results of in vitro fertilization attempts in women 40 years of age and older: The Norfolk experience. Fertil Steril 1987; 47: 130-137.

52. Navot D, Rosenwaks Z, Margalioth EJ. Prognostic assessment of female fecundity. Lancet 1987; 19: 645-651.

53. Toner JP, Philput CB, Jones GS, Muasher SJ. Basal follicle stimulating hormone levels is a better predictor of in vitro fertilization performance than age. Fertil Steril 1991; 55: 784-791.

54. Muasher SJ, Oehninger S, Simonetti S. The value of basal and/or stimulated serum gonadotropin levels in the prediction of stimulation response and in vitro fertilization outcome. Fertil Steril 1988; 50: 298-307.

55. Fanchin R. De Ziegler D, Olivennes. Exogenous follicle stimulant hormone ovarian reserve test (Efort): a simple a reliable screening test for detecting poor responders in vitro fertilization. Human Reprod 1994; 9: 1607-1611.

56. Richard T, Scott Jr MD. Evaluation and treatment of low responders. Sem Reprod Endocrinol 1996; 14: 317-337.

57. Scott RT, Leonardi MR, Hoffman GE. Prospective evaluation of clomiphene citrate challenge test screening in the general infertility population. Obstet Gynecol 1993; 82: 539-545.

58. Seifer DB, Lambert-Messerlian G, Hogan JW, Gardiner AC, Blazar AS, Berk CA. Day 3 serum inhibin-B is predictive of assisted reproductive technologies outcome. Fertil Steril 1997; 67: 110-114.

59. Balash J, Creus M, Fabregues F, Carmona F. Inhibin, follicle stimulant hormone, and age as predictors of ovarian response in invitro fertilization cycles stimulated with gonadotropin- realizing hormone agonist-gonadotropin treatment. Am J Obstet Gynecol 1996; 175: 1226-1230.

60. Loumaye E, Billion JM, Mine JM. Prediction of individual response to controlled ovarian hyperestimulation by means of clomiphene citrate challenge test. Fertil Steril 1990; 53: 295-301.

61. Levy T, Limor R, Villa Y, Eshel A, Eckstein N, Vagman I, Lidor A, Ayalon D. Another look at co-treatment with growth hormone and human menopausal gonadotropins in poor ovarian responders. Human Reprod 1993; 6: 834-849. 62. Battaglia DE, Goodwing P, Klein NA, Soules MR. Influence of maternal age on meiotic spindle assembly in oocytes from naturally cycling women. Human Reprod 1996; 11: 2217-2222.

63. Benadiva AC, Kleigman I, Rosenwaks Z. Assisted Reproductive Technology versus tubal surgery in Operative Gynaecology Endoscopy. Joseph S, Sanfilipo and Ronald L Levine, editor. 1989; 278-305.

64. Navot D, Bergh PA, Williams MA. Poor oocyte quality rather than implantation failure is a cause of age related decline in female infertility. Lancet 1991; 337: 1375-1381. 65. Munne S, Alikani M, Tomkin G. Embryo morphology, developmental rates and maternal age are correlated with chromosome abnormalities. Fertil Steril 1995; 64: 382-389.

66. Padilla SL, García JE. Effect of maternal age and number of in vitro fertilization procedures on pregnancy outcome. Fertil Steril 1989; 52: 270-276.

67. Mage G, Pouly JL, Bouquet de Joliniere J, Chabrand J, Riouallon A, Bruhat MA. A preoperative classification to predict the intrauterine and ectopic pregnancy rates after distal tubal microsurgery. Fertil Steril 1986; 46: 807-810. 68. Dubuisson JB, Chapron C, Morice P, Aubriot FX, Foulot H, Bouquet de Joliniere J. Laparoscopic salpinguectomy fertility results according to the tubal mucosal appearance. Human Reprod 1994; 9: 334-339.

69. Singhal V, Li TC, Cooke ID. An analysis of factors that influencing the outcome of 232 consecutives tubal microsurgery cases. Br J Obstet Gynaecol 1991; 98: 628636.

70. Meyer WR, Beyler SA. Deleterious effects of hidrosalpingues on in vitro fertilization and endometrial integrin expression. Ass Reprod Rev 1995; 5: 201-203. 